\title{
Microwave Dielectric Characterization of Carbon Nanotube Networks
}

\author{
Emmanuel Decrossas and Samir M. El-Ghazaly \\ University of Arkansas, 3217 Bell Engineering Center, Fayetteville, AR,
}

USA

\section{Introduction}

Carbon nanotubes (CNTs) have been discovered in the early 1990s (Iijima, 1991). The earliest research has been focus on the synthesis of CNTs to produce sufficient amount of material necessary for experiments. The progress of common growth techniques including arc discharge and laser ablation of graphite pieces and later chemical vapor deposition (CVD) and plasma enhanced CVD has significantly improved the quantity as well as the quality of the CNTs (Meyyapan, 2005). In the last decade many companies have concentrated their efforts to develop and control those growth techniques to insure very high purity and uniform CNTs in length and diameter at a reasonable cost. The physical properties of carbon nanotubes (Saito, 1998) have demonstrated unique electronic structure depending on their dimensions and chiralities, which have been considered as a major discovery in semiconductor devices. In addition, due to strong covalent carbon-carbon bonding CNTs present very high tensile strength, thermal and electrical conductivity. Hence, those mechanical and electrical properties make them also very attractive to reinforce composites material for aerospace applications. Usually, carbon nanotubes are classified in two categories: Single-wall CNTs (SWCNT) composed of a single graphitic cylinder where the diameter varies from 0.7 to $2 \mathrm{~nm}$ and Multiwall CNTs (MWCNTs) composed of several concentric graphitic layers where the diameter varies from 10 to $200 \mathrm{~nm}$. Commercial carbon nanotubes are available in a powder form where they are entangled and randomly distributed.

Today, the growth of carbon nanotubes is well advanced and the main research interest has become CNT-based applications. The potential of carbon nanotube-based applications is currently investigated for developing novel transistors, scanning probes (M.J. O'Connell, 2006), mechanical sensors (Tung et al., 2007), field emission and lighting applications (Jorio et al., 2008). The knowledge of dielectric properties at low frequency (DC to $1 \mathrm{MHz}$ ) as well as high frequency (above $1 \mathrm{MHz}$ ) is the key for developing novel electronic devices such as radio frequency miniaturized resonators (El Sabbagh and El-Ghazaly, 2009a) as well as electronic model for future implementation (EL Sabbagh and El-Ghazaly, 2009c). Therefore, the electrical characterization at radio frequency/microwave $(\mathrm{RF} / \mathrm{M})$ bands becomes a challenge where common measurement techniques are inappropriate. For high frequency measurements, complex permittivity $\left(\varepsilon=\varepsilon^{\prime}-j \varepsilon^{\prime \prime}\right)$ is usually used to describe the dielectric properties of a material where the real part of the permittivity is related to the dielectric constant and the imaginary part can be associated to the conductivity. 
Over 600 publications have reported the characterization of CNT-based composites in 2010 where carbon nanotubes are embedded in a host medium. However, those samples preparation considerably decreases the accuracy of the measurements and explains the difference between the reported results. In this chapter, a non destructive, low-cost, easily implemented and broad frequency band (from $10 \mathrm{MHz}$ to $50 \mathrm{GHz}$ ) measurement setup is presented where only a small fraction of material in a powder form is necessary (Decrossas et al., 2010). Carbon nanotubes powder as furnished by the supplier is placed without host medium decreasing the unknown effects on the preparation of the samples. The extraction of the complex permittivity of CNT networks is investigated over a wide frequency band considering different powder densities. Then, the reported results are explained through the percolation theory (Stauffer and Aharony, 1992) as well as numerical and experimental studies where metallic particles are embedded in a dielectric medium (Decrossas et al., 2011). The conclusion summarizes the experimental reported results and is followed by a discussion on possible future research directions.

\section{Description of the test structure}

The measurement technique is realized by studying the propagation of electromagnetic waves in a circular waveguide shorted at the end filled with a dielectric medium. Therefore, incident electromagnetic waves are reflected after propagating in a medium and the study of these waves inform us about the electrical properties of the material under test (MUT).

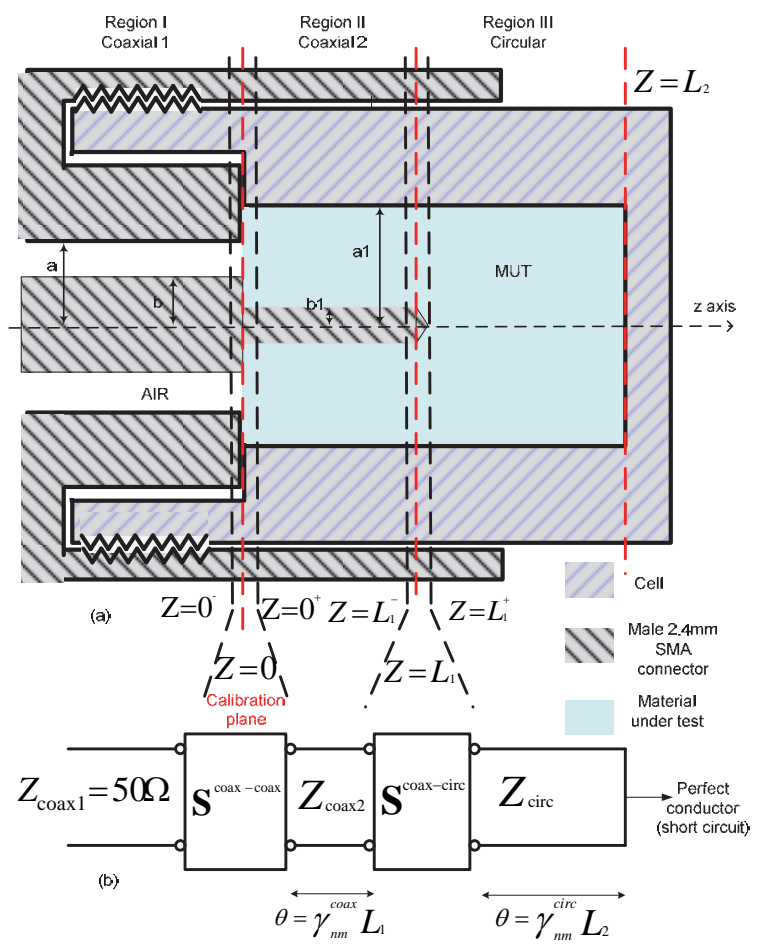

Fig. 1. (a) Schematic of the test structure. (b) Generalized scattering matrices building blocks. 
The homemade test structure includes coaxial and circular discontinuities where the material under test filled the circular waveguide as shown in Fig. 1.The measurements are carried out using a performance network analyzer (PNA) capable of measurements from 10 $\mathrm{MHz}$ to $67 \mathrm{GHz}$. The hollow circular waveguide is connected to the PNA through a precision adapter via $1.85 \mathrm{~mm}$ coaxial cable. Copper well-known for its high conductivity has been chosen to build the cell holding the material under test as depicted in Fig. 2.

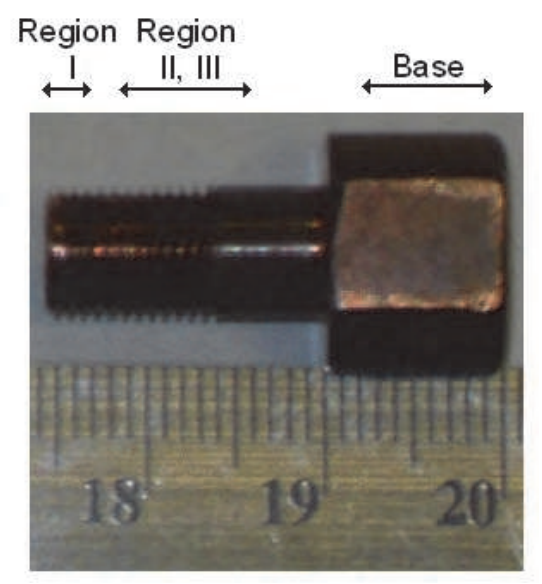

Fig. 2. Actual picture of the fabricated testing structure. Regions I, II, and III are the same as those shown in the schematic of Fig. 1. Dimensions of design parameters shown in Fig. 1 are: $\mathrm{a}=1.2 \mathrm{~mm}, \mathrm{~b}=0.52 \mathrm{~mm}, \mathrm{a}_{1}=1.26 \mathrm{~mm}, \mathrm{~b}_{1}=0.254 \mathrm{~mm}, \mathrm{~L}_{1}=1.1 \mathrm{~mm}, \mathrm{~L}_{2}=5.3 \mathrm{~mm}$.

The adopted measurement technique presents several advantages: (a) only a small fraction of carbon nanotubes powder is required; (b) other parameters like density and temperature are easily controlled for repeatable measurement; (c) the powder is held in a closed structure to avoid fringing radiation. The extraction technique includes the computation of the reflection coefficient using the mode matching method where each discontinuity encounter by an incident wave is characterized by its generalized scattering parameters as shown in Fig. 1(b). The accuracy of the analysis comes from the consideration of all propagating and evanescent higher-order modes excited by the discontinuities. The model of the test structure using the mode matching technique (MMT) and the methodology to extract the complex permittivity have been previously described (Decrossas et al., 2011). If the problem to derive the reflection coefficient from the full-wave model is straightforward, the computation of the complex permittivity is obtained by an iterative method. An optimized gradient method solves this inverse problem based on comparison of the measured and simulated reflection coefficient using the mode matching technique.

In case of material in dry-powder form, density is an important parameter that affects the value of complex permittivity. The procedure to extract the permittivity of CNT networks involves also the study of the effect of the density of CNT networks inside the waveguide holder (Decrossas et al., 2011). This is carried out by increasing gradually the mass of added networks inside the holder. To calibrate the measurement setup and verify the method of extraction, experiments are first realized with silicon dioxide $\left(\mathrm{SiO}_{2}\right)$ as well as pure alumina powder $\left(\mathrm{Al}_{2} \mathrm{O}_{3}\right)$ with particles size of approximately $50 \mathrm{~nm}$. Fig. 3 presents the real and 


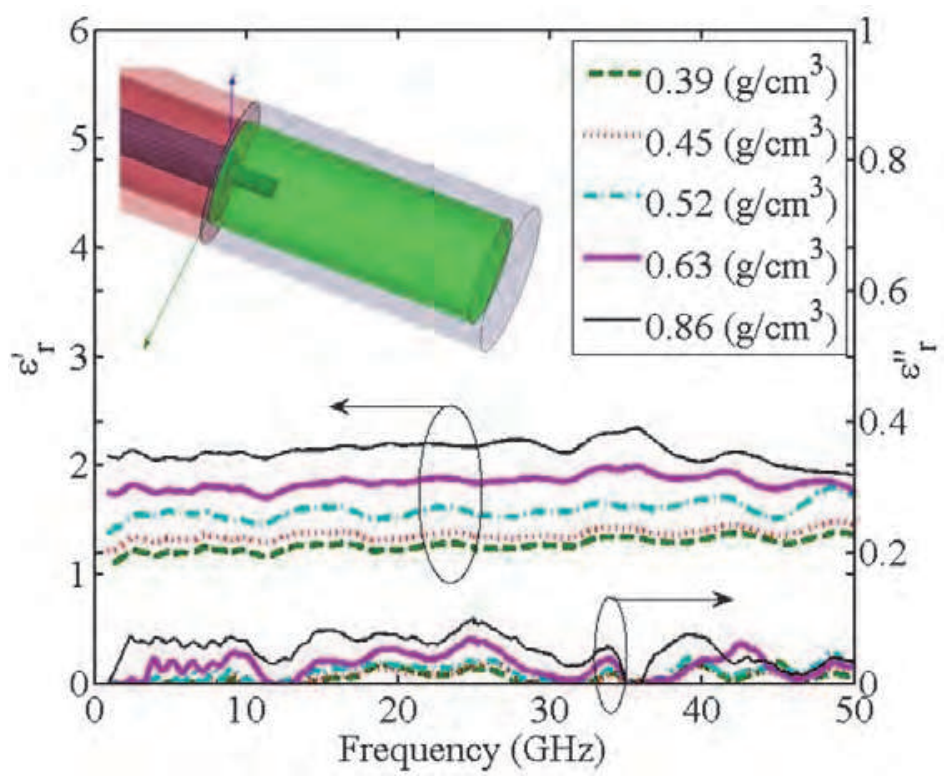

Fig. 3. Variation of extracted effective permittivity of pure pulverized $\mathrm{SiO}_{2}$ versus frequency using the test setup as shown in Fig. 1.

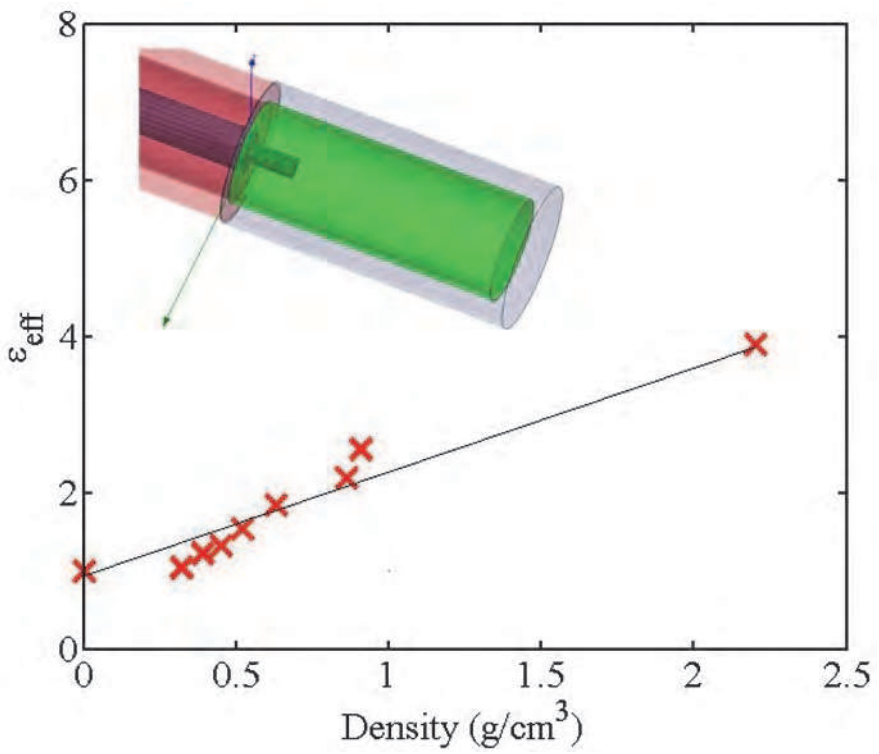

Fig. 4. Variation of extracted effective permittivity of pure $\mathrm{SiO}_{2}$ versus density realized at frequency of $3 \mathrm{GHz}$. The last point is the common accepted value for $\mathrm{SiO}_{2}, \varepsilon_{\text {eff }}=3.9$ for a density of $2.2 \mathrm{~g} / \mathrm{cm}^{3}$. 
imaginary part of the extracted complex relative permittivity for $\mathrm{SiO}_{2}$ powder for various packing densities considering the test setup shown in Fig. 1. Fig. 4 shows the variation of the extracted real part of the effective permittivity of silicon dioxide function of the density at 3 GHz.

The density is computed by weighting the powder using an analytical balance divided by the known volume of the hollow circular waveguide $\left(\rho=\frac{w}{V} g / \mathrm{cm}^{3}\right)$. Then the density is gradually increased by packing the powder inside the holder using a manual press. It exists different formulations to estimate the permittivity of bulk material from its pulverized form (Nelson, 1992). However, those formulations are more appropriate for particle size of 50$100 \mu \mathrm{m}$. From experimental data the variation of the effective permittivity with the packing density for nano-particles suggests a linear dependence instead as shown in Fig. 4 and Fig. 6. Fig. 5 shows the variation of the real part of the effective permittivity of pure alumina powder $\left(\mathrm{Al}_{2} \mathrm{O}_{3}\right)$ for various densities at low frequency up to $3 \mathrm{Ghz}$, it is interesting to observe the flat curves in those cases.

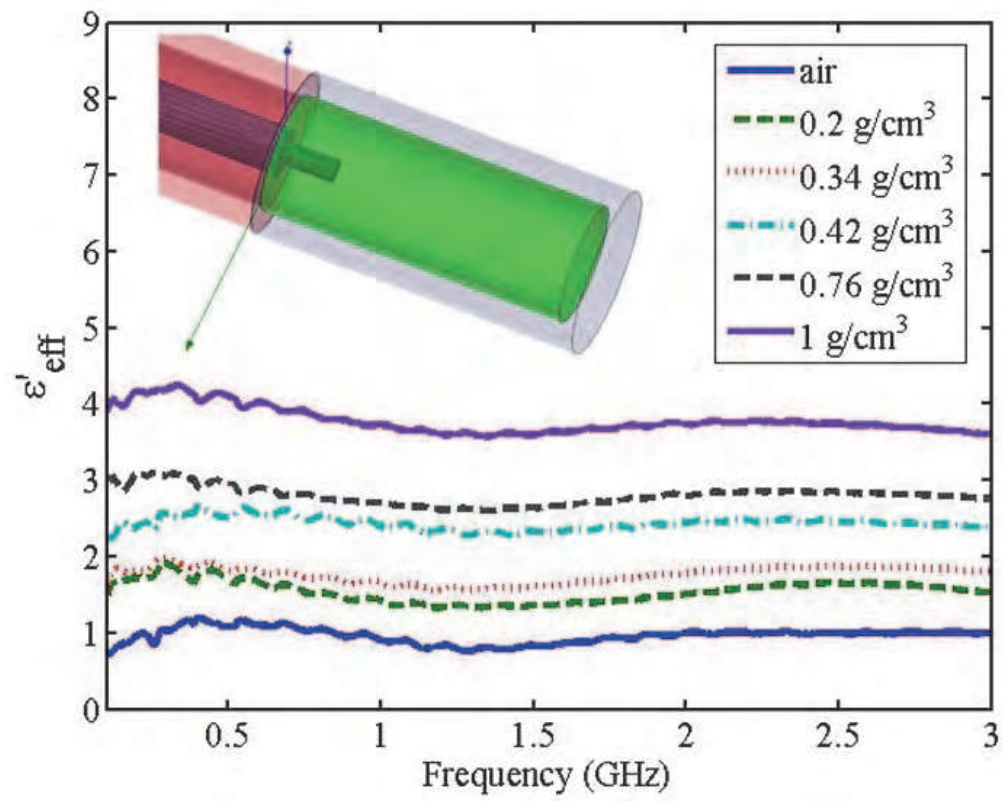

Fig. 5. Variation of extracted effective dielectric constant of pure $\mathrm{Al}_{2} \mathrm{O}_{3}$ powder versus frequency at different densities using the test setup shown in Fig. 1.

Similarly to the case of silicon dioxide in Fig. 4 the linear variation of the real part of the permittivity of pure alumina powder is presented in Fig. 6. The usual formulations to compute the bulk permittivity from pulverized material doesn't seem appropriate in case of nano-particles and overestimate the common reported value for bulk material. We have used dielectric material with low loss and well known properties to study the variation of the real part of the effective permittivity and provide a reference study to compare with the microwave characterization of carbon nanotube powder. The variation of the real part of the 
effective permittivity of composites material with densities is compared with the results shown in Fig. 6 to support our experimental studies.

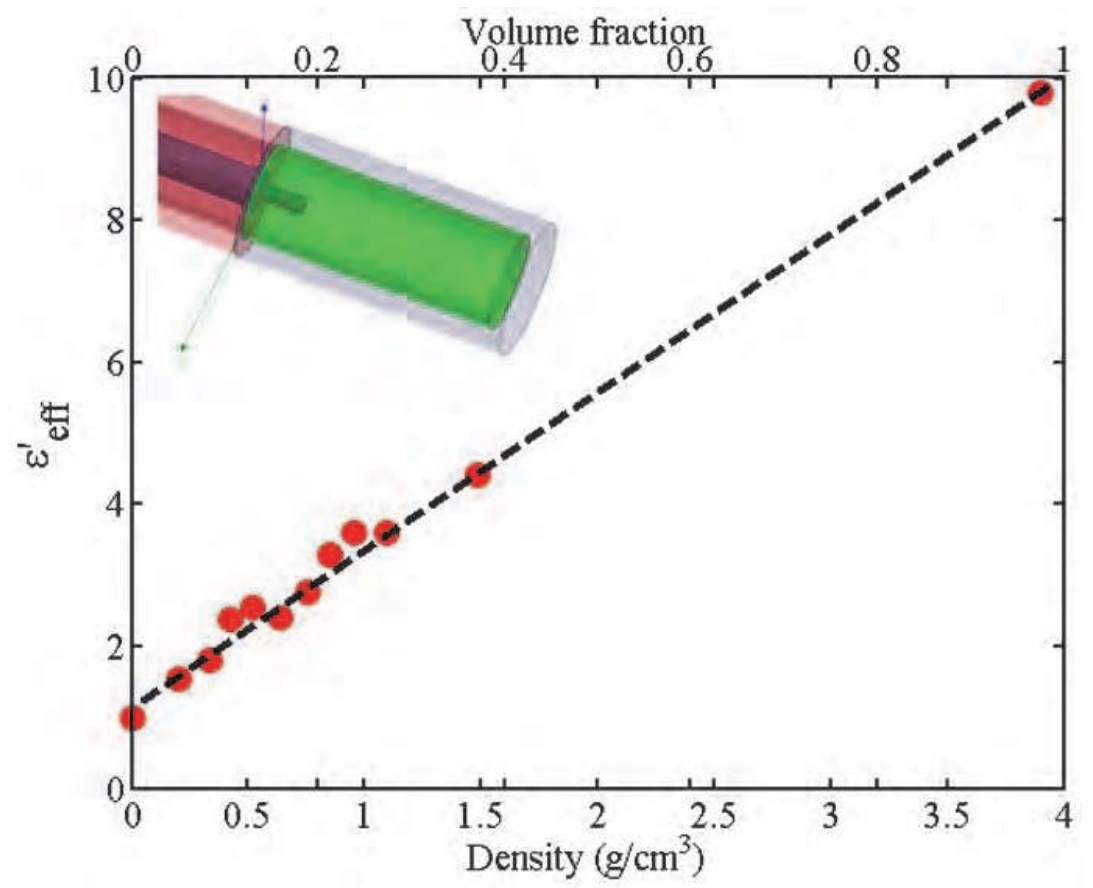

Fig. 6. Variation of extracted effective dielectric constant of pure $\mathrm{Al}_{2} \mathrm{O}_{3}$ versus density or the volume fraction at $3 \mathrm{GHz}$ using the test setup shown in Fig. 1. The last point is the common accepted value $\varepsilon_{\text {eff }}^{\prime}=9.8$ at a bulk density of $3.9 \mathrm{~g} / \mathrm{cm}^{3}$ for pure alumina.

\section{Complex permittivity of CNT network}

Similarly it is possible to extract the complex effective permittivity of carbon nanotubes powder as furnished by the manufacturer (Sigma-Aldrich). The powder purity is 50 to 70 volume percentage as determined by Raman spectroscopy and scanning electron microscopy (SEM) and contains residual catalyst impurities nickel and yttrium. The lengths of CNTs are approximately $20 \mu \mathrm{m}$. In house SEM is carried out and it shows the presence multi-walled carbon nanotubes as well as single-walled CNTs within the sample. Fig. 7 to Fig. 10 present the variation of the real and imaginary part of the relative complex permittivity $\left(\varepsilon_{r}=\varepsilon_{r}^{\prime}-j \varepsilon_{r}^{\prime \prime}\right)$ over a broad frequency band (10 Mhz to $\left.50 \mathrm{Ghz}\right)$ for different densities (Decrossas et al., 2011).

The highest value of the real part of the relative complex permittivity obtained at low frequency is $1.6 \times 10^{5}$ and decreases continuously to finally reach 4.5 at $50 \mathrm{GHz}$. Similarly, the highest value of the imaginary part of the relative complex permittivity shown is $4.1 \times 10^{3}$ and decreases to 5 at $50 \mathrm{GHz}$. Fig. 7 is plotted on a log scale due the fast variation of the relative permittivity at low frequency. Then at high frequency the variation presented in Fig. 
8 is reduced and suggests asymptotic values. The different scales highlight the frequency dependence of the relative complex permittivity of CNT networks. Similarly, the imaginary part of the relative permittivity is illustrated in Fig. 9 and Fig. 10.

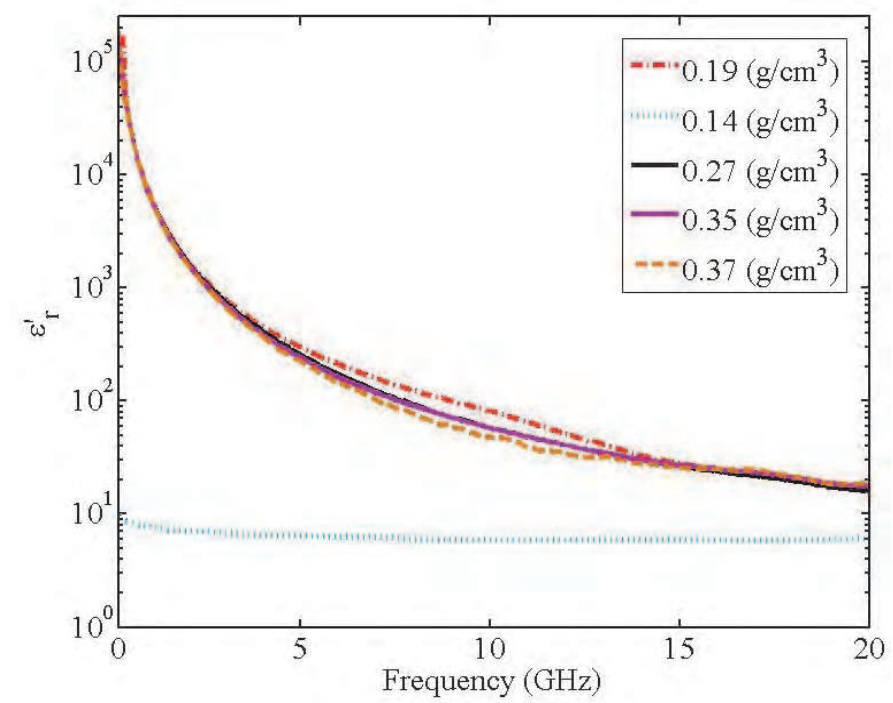

Fig. 7. Variation of the real part of the relative permittivity of carbon nanotubes networks for different densities, at high frequencies.

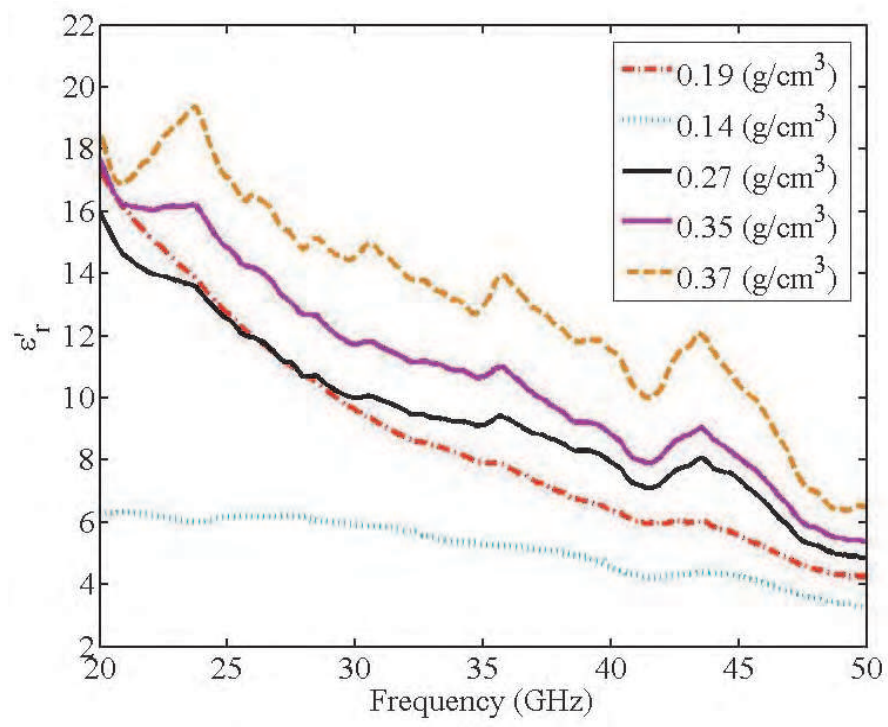

Fig. 8. Variation of the real part of the permittivity of carbon nanotubes networks at low frequencies considering various densities. 


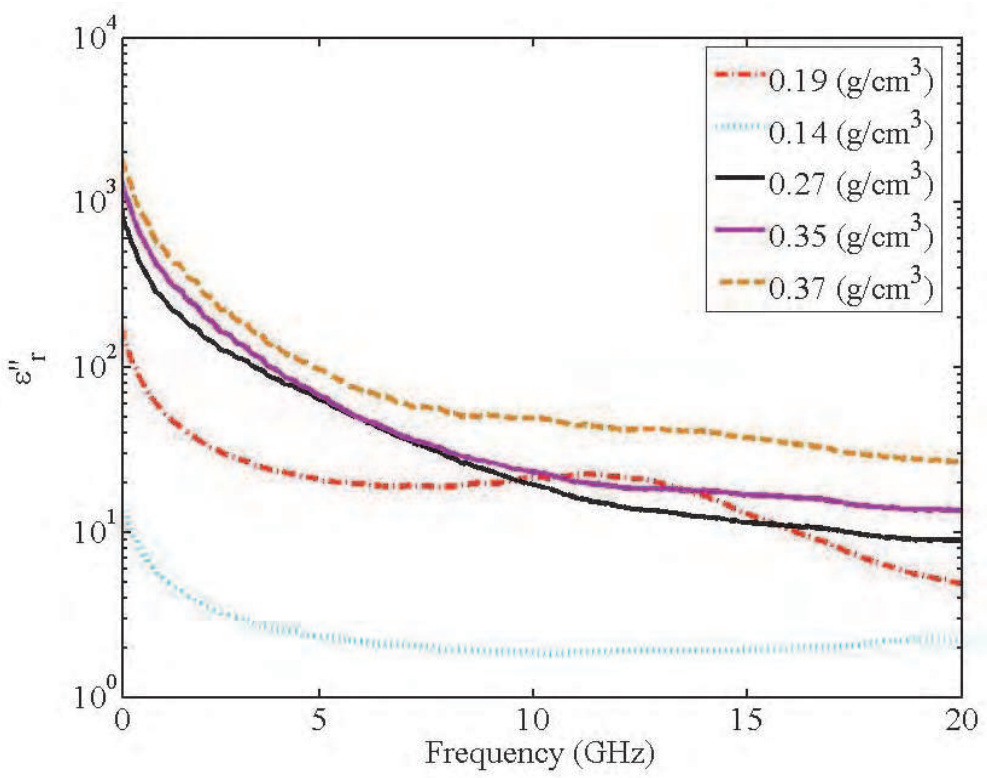

Fig. 9. Variation of the imaginary part of the relative permittivity of carbon nanotubes networks for different densities, at low frequencies.

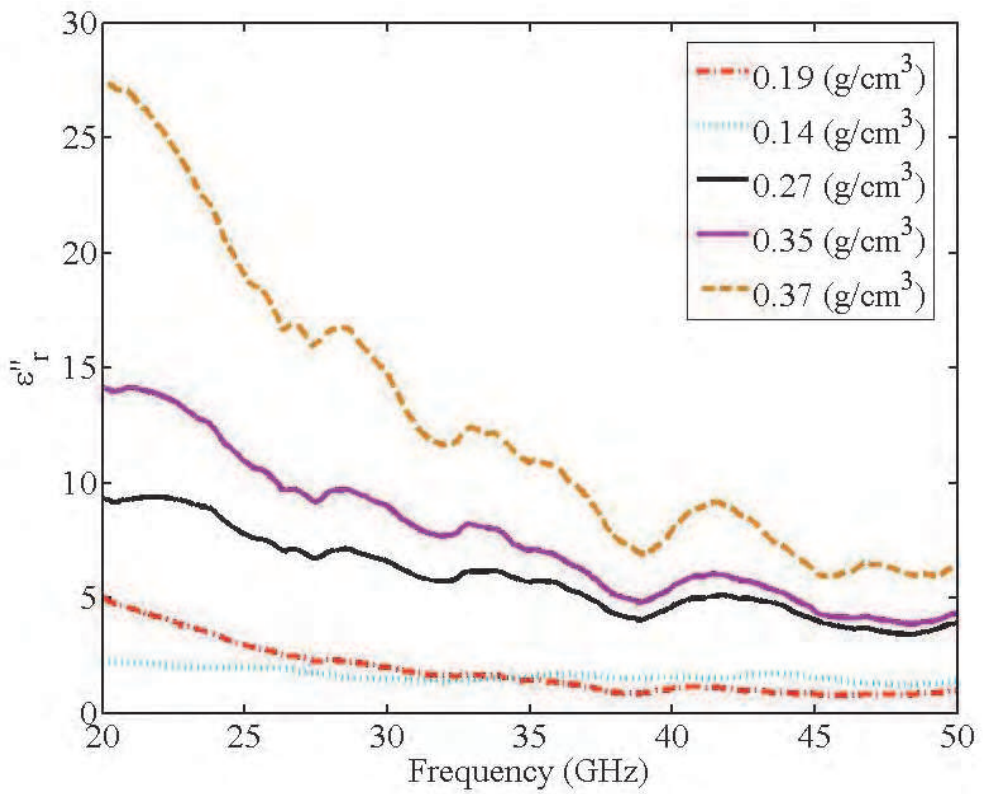

Fig. 10. Variation of the imaginary part of the permittivity of carbon nanotubes networks at high frequencies for various densities. 


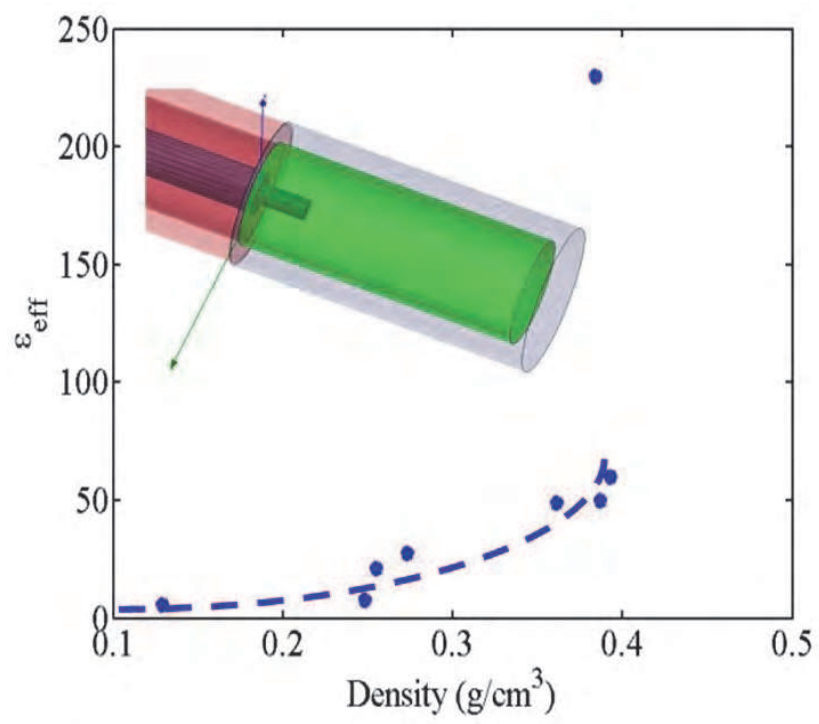

Fig. 11. Variation of the real part of the effective permittivity of carbon nanotube networks function of the density at $10 \mathrm{GHz}$.

The complex relative permittivity is continuously increasing versus the density of CNT networks as shown in Fig. 11 following the percolation theory (Stauffer and Aharony, 1992). The values obtained at high frequency are in agreement with the values reported (N.N. Al Moayed et al., 2007). Moreover, the large values at low frequency are also consistent with those large values reported (M.A. EL Sabbagh et al., 2009b, and H. Xu et al., 2007).

It should be noted the curve difference of the real part the permittivity function of the density between Fig. 11 and Fig. 4, Fig. 6. The percolation theory may provide a physical explanation about the unusual very high values of the dielectric properties. The high values obtained for the imaginary part of the complex relative permittivity can be attributed to high conductivity (Ebbensen et al., 1996) of carbon nanotubes using the following relation $\varepsilon_{r}^{\prime \prime}=\frac{\sigma}{\omega \varepsilon_{0}}$ where $\sigma$ is the conductivity of CNT and $\omega=2 \pi f$ the frequency relation.

The very large values of the real part of the permittivity are explained in the next section where metallic particles are embedded in a dielectric medium and the complex effective permittivity of the mixture is experimentally extracted (Decrossas et al., 2011b).

\section{Complex permittivity of composites material by mixing metallic particles in dielectric medium}

\subsection{Finite element simulation}

To study the variation of the dielectric constant, we have randomly embedded metallic spheres in a fictive dielectric $\left(4.5-j^{*} 0.88\right)$. The metallic spheres considered as perfect electrical conductor have a radius of $0.5 \mathrm{~mm}$ and are randomly positionned in the dielectric. Then, the effective permittivity is extracted using the mode matching method. The simulations are realized using the 3D full-wave modeling software HFSSTM by Ansoft. 
It should be noted that MMT considers the material under test as an homogenous medium while the finite element method considers the interactions between the metallic spheres. For this reason we are only able to extract the effective complex permittivity of the mixture. In addition, by increasing the number of randomly positionned metallic spheres in the host medium considerably augment the computing simulation time and as long as 3 days are necessary to run the last simulation presented in Fig. 12. Those simulations even if the metallic balls dimensions cannot be compared to nano-particles are necessary to demonstrate the effect of the metallic particles in a dielectric medium.

By embedding metallic spheres in the medium, HFSS simulations present an enhancement of the effective dielectric constant as presented in Fig. 12. The extraction of the complex permittivity in addition to the comparison between the finite element method and the mode matching technique validate the process of electrical characterization of the material under test.

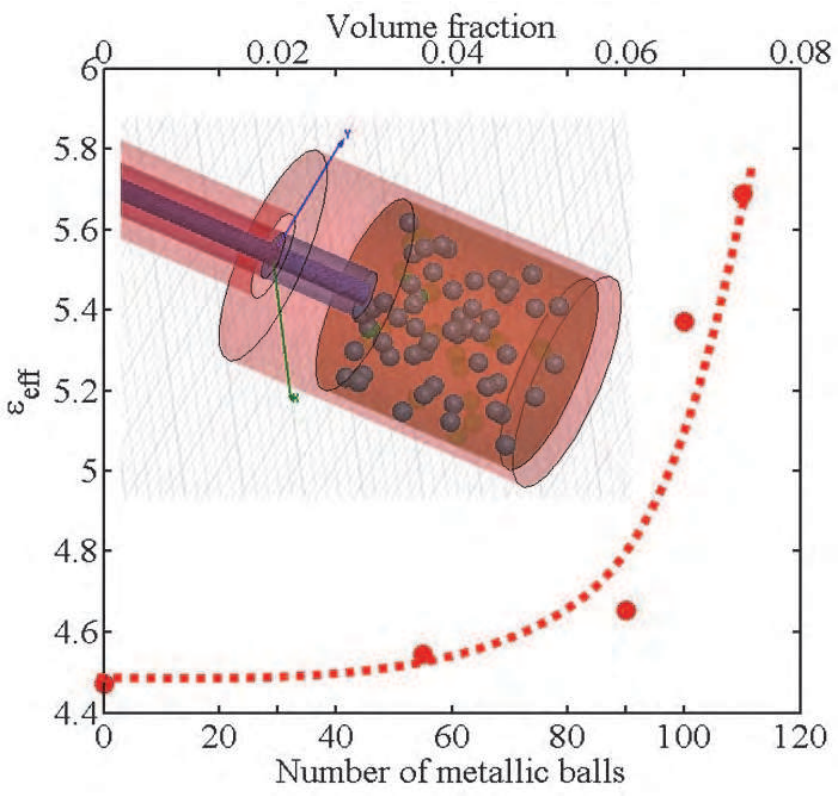

Fig. 12. Variation of the simulated effective permittivity using HFSS by increasing the spherical metallic particles in a fictive dielectric host medium.

\subsection{Extracted complex permittivity of copper mixed with alumina particles composites material}

The percolation behavior is verified experimentally by embedding metallic particles in a dielectric medium. This theory is usually applied to model the change of material where the increase of metallic particles creates some sort of conductive paths through the dielectric material. In other words, below the percolation threshold the material keeps behaving as a dielectric until the critical concentration point called percolation threshold is reached and the material becomes conductor. This phenomenon occurs at low frequency where the electromagnetic wavelength is larger than the interstice between metallic particles. So, we have concentrated our efforts to extract the effective complex permittivity up to $3 \mathrm{GHz}$. A similar behavior exists in carbon nanotube networks where both conducting and 
semiconducting CNTs as well as metal catalyst impurities are present. This work shows how embedded metallic particles enhance the effective relative permittivity of carbon nanotubes bundles due to metallic particle interactions in the dielectric medium below percolation threshold, providing a physical explanation on the usually reported very high value of the complex permittivity. The enhancement of the effective permittivity is realized by mixing copper particles with dry powder of alumina particles. The sketch in Fig. 13 illustrates the realized mixture which is dropped and packed in our setup to extract the effective permittivity of the mixture for different densities and concentrations.

The copper powder as furnished by the manufacturer (Alfa Aeser) has $99 \%$ purity with particle sizes varying between $0.5-1.5 \mu \mathrm{m}$. Two different weight percentages of copper mixed with alumina powder is studied to show the enhancement of the real part of the permittivity. (a) Copper has $75 \%$ weight obtained by mixing $0.5 \mathrm{~g}$ of Alumina with $1.5 \mathrm{~g}$ of Copper and (b) copper has $20 \%$ weight achieved by mixing $1 \mathrm{~g}$ of Alumina with $0.25 \mathrm{~g}$ of Copper.

It should be noted that copper particles tend to oxidize with air contact, consequently a thin layer of oxide copper $(\mathrm{CuO})$ coats copper $(\mathrm{Cu})$ particles. Fig. 14 illustrates the variation of the extracted complex effective function of the frequency for the $75 \%$ weight mixture.

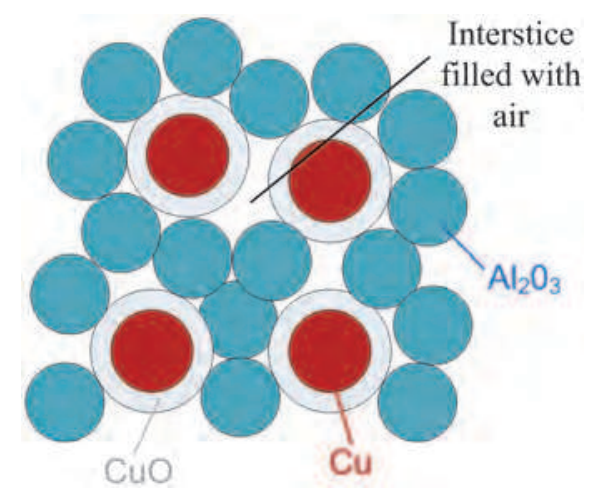

Fig. 13. Illustration of the realized composite material under test (not to scale). The size of the alumina $\left(\mathrm{Al}_{2} \mathrm{O}_{3}\right)$ particles is $0.05 \mu \mathrm{m}$ while the size of the copper $(\mathrm{Cu})$ particles is $0.5-1.5 \mu \mathrm{m}$.

It should be noted that in Fig. 14, an enhancement of the real part is observed while the imaginary part related to the conductivity is still low which is characteristic of a dielectric composite material with a composition below percolation threshold.

Two mechanisms may explain this permittivity enhancement (Fiske et al., 1997, and Ravindran et al., 2006): a) the electrical field creates a surface charge polarization on metallic particles at the dielectric interface which yields an increase in capacitance. b) The dipole polarization contributes to the global permittivity when the electrical field frequency is slower than the relaxation frequency of the metallic particles. This phenomenon occurs at low frequency when the wavelength is larger than the metallic-semiconductor-metallic interactions. Fig. 15 presents a comparison of the extracted real part of the effective permittivity function of the density, highlighting the enhancement of the dielectric constant compared to pure alumina powder as reported in Fig. 6.

All those experiments have been realized considering spherical alumina and copper particles. However, some studies suggest that the shape of the metallic particles also influence the behavior of the effective permittivity (Link et al., 1999, and Guo et al., 2010). To illustrate this idea the next section discusses the mixture of alumina powder with carbon nanotubes. 


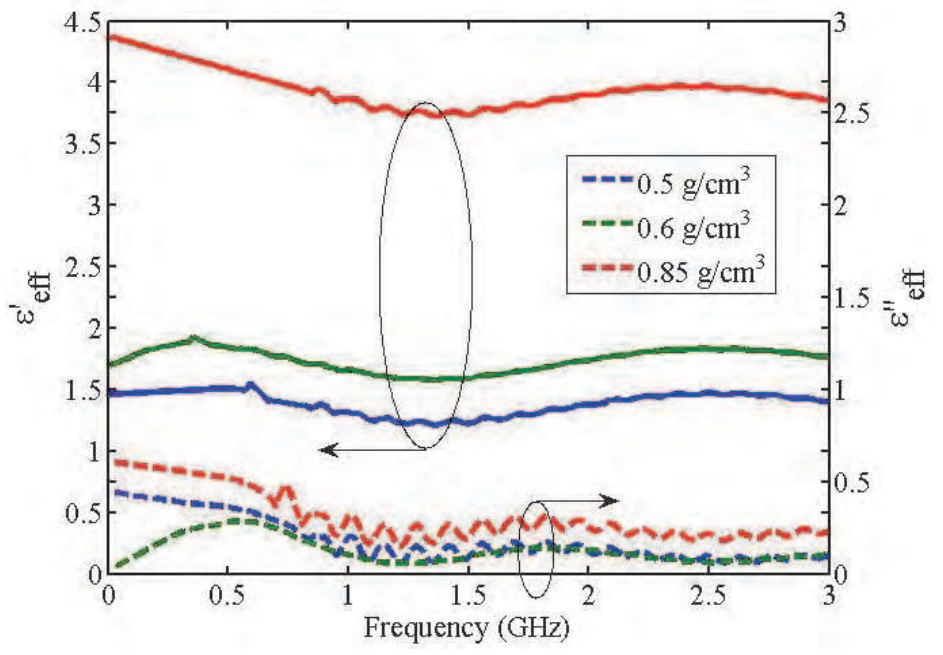

Fig. 14. Extraction of the complex effective permittivity $\left(\varepsilon_{\text {eff }}=\varepsilon_{\text {eff }}^{\prime}-j \varepsilon_{\text {eff }}^{\prime \prime}\right)$ considering the $75 \%$ weight mixture of Alumina-copper for different densities functions of the frequency using the test setup shown in Fig. 1.

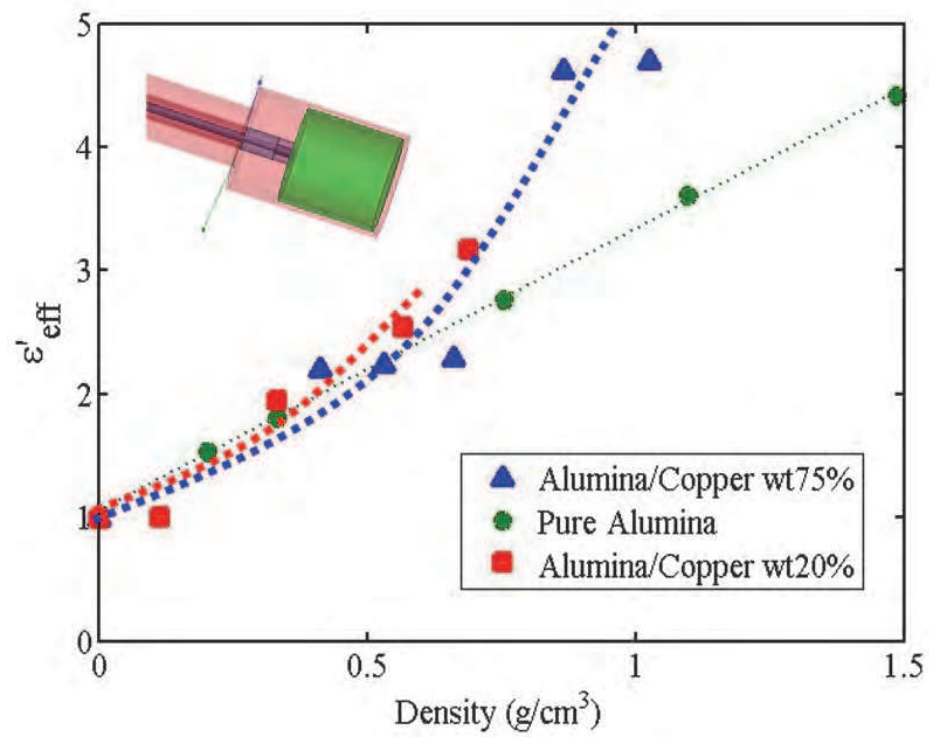

Fig. 15. Variation of the effective permittivity of the composite material function of the density realized at $60 \mathrm{MHz}$ and compared to pure alumina powder.

\section{Complex permittivity of CNT-based composites material}

The enhancement and the frequency dependence of the permittivity due to the presence of carbon nanotubes embedded in a dielectric medium are illustrated experimentally 
considering different concentrations and packing densities. Those experiments show the possibility to engineer novel composite materials by tuning the electrical response of this material under an electrical field (Liu et al., 2007). Controlling the dispersion, the size, the shape of those particles into the medium are the key of engineering those new composite materials. Fig. 16 is a picture of the prepared mixture of alumina and carbon nanotubes using a scanning electron microscope (SEM).

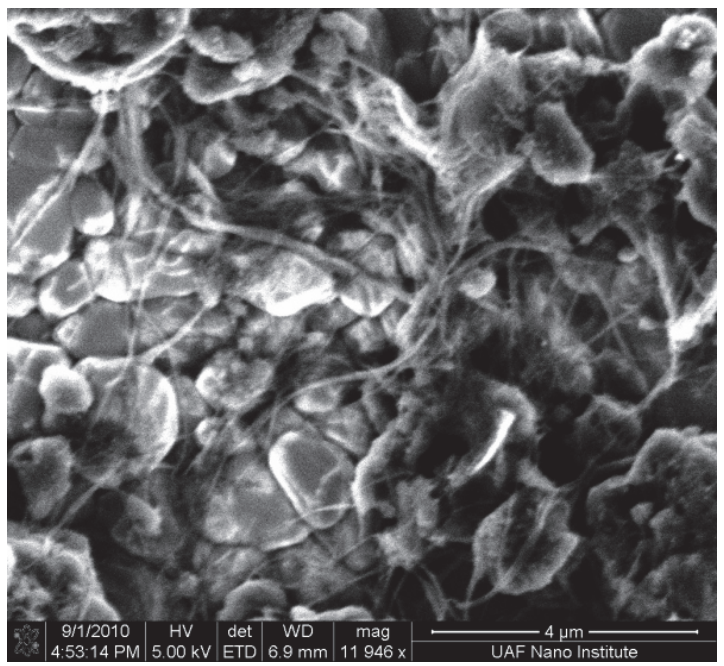

Fig. 16. SEM picture of the prepared mixture of alumina and carbon nanotubes.

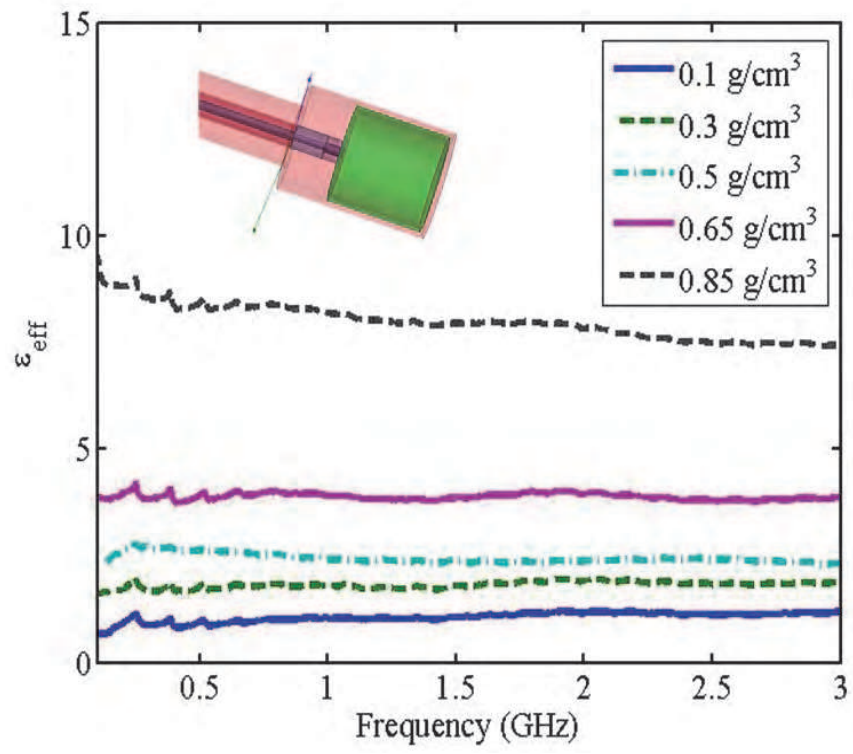

Fig. 17. Variation of extracted real part of the effective permittivity of carbon nanotubes mixed with alumina versus frequency using the test setup as shown in the inset. 
The CNT-based composite material is realized by mixing $1 \mathrm{~g}$ of alumina with $0.2 \mathrm{~g}$ of carbon nanotubes powder. Fig. 17 illustrates the enhancement of the real part of the effective permittivity at various packing densities. It should be noted that at low density the variation of the effective permittivity shows a flat response indicating a low frequency dependence of the dielectric constant. However, when the density is $0.85 \mathrm{~g} / \mathrm{cm}^{3}$ in the hollow circular waveguide the dielectric constant decreases with the frequency. As explained in the previous section the permittivity enhancement at low frequency is due to the interfaces between the metallic and dielectric particles. When the frequency increases, i.e. the wavelength decreases, this phenomenon disappears and explains the slope of the dielectric plot at high density (black dashed curve in Fig. 17).

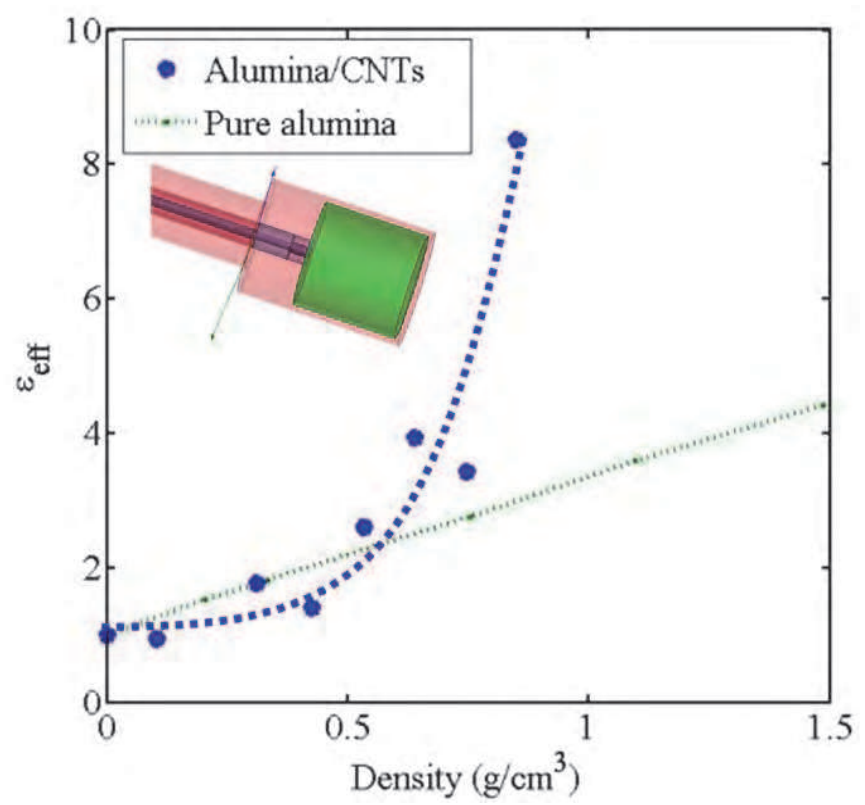

Fig. 18. Variation of the real part of the effective permittivity of CNTs mixed with alumina powder versus the density realized at $60 \mathrm{MHz}$. The variation of the real part of the effective permittivity for pure alumina powder with density is provided as a comparison as reported in Fig. 6.

\section{Conclusions}

The electrical characterization at $\mathrm{RF} /$ microwave frequencies is the key to understand and model devices for future implementation. For this purpose, the frequency dependence of the effective permittivity for carbon nanotubes powder as well as CNT-based composites materials has been extracted. In this chapter, the frequency dependence of the complex permittivity has been demonstrated over a broad frequency band using only one testing setup. The carbon nanotubes powder is analyzed without samples preparation increasing the accuracy of the measurements. It appears that the microwave properties are sensitive to the samples preparation of composite materials due the dispersion of the metallic particles into the host medium. The experiments on composite material where metallic particles are 
mixed with a dielectric medium provide an explanation about the high value of the effective permittivity obtained for carbon nanotubes powder. The effective permittivity enhancement is explained based on the percolation theory caused by the multiple metallic-dielectric interfaces in the medium encountered by incident waves. The various results suggest that CNT-based composite material may reduce the size of radio frequency/microwave (RF/M) devices by engineering the dielectric constant of the substrate. The tunability of the real and imaginary part independently may have a great potential in novel RF/M devices such as smart material as well as dispersive media for electromagnetic shielding.

\section{Acknowledgements}

The authors thank M.A EL Sabbagh (Univ. of Syracuse, USA), V. Fouad Hanna (Univ. Pierre et Marie Curie-Paris 6, France), and H.A. Naseem (Univ. of Arkansas, Fayetteville) for fruitful conversations on this topic, D. Rogers for the fabrication of the test structure and M. Benamara for his experience about the preparation of SEM samples. This research was sponsored by the Army Research Laboratory and was accomplished under Cooperative Agreement Number W911NF-10-2-0072. The views and conclusions contained in this document are those of the authors and should not be interpreted as representing the official policies, either expressed or implied, of the Army Research Laboratory or the U.S. Government. The U.S. Government is authorized to reproduce and distribute reprints for Government purposes notwithstanding any copyright notation herein.

\section{References}

Al Moayed, N.N., Khan U.A., Obol, M., Gupta, S., \& Afsar, M.N. (2007). Characterization of single- and multi-walled carbon nanotubes at microwave frequencies, Proceedings of Instrumentation and Measurement Technology Conference, pp. 1-4, ISBN 1-4244-0588-2, Warsaw, Poland, May 1-3, 2007.

Decrossas, E., EL Sabbagh, M.A., Fouad Hanna, V., \& El-Ghazaly, S.M. (2010). Broadband characterization of carbon nanotube networks, in IEEE International Symposium on Electromagnetic Compatibility, pp. 208-211, ISBN 978-1-4244-6305-3, Fort Lauderdale, Florida, USA, July 25-30, 2010.

Decrossas, E., EL Sabbagh, M.A., Fouad Hanna, V., and El-Ghazaly, S.M. (2011). Rigorous Characterization of Carbon Nanotube Complex Permittivity over a Broadband of RF Frequencies, in IEEE Electromagnetic Compatibility Trans., under review.

Decrossas, E., EL Sabbagh, M.A., Naseem, H.A., Fouad Hanna, V., \& El-Ghazaly, S.M. (2011b) Effective Permittivity Extraction of Dielectric Nano-powder and Nano-composite Materials: Effects of Packing Densities and Mixture Compositions" in IEEE European Microwave Week, Manchester, UK, October 9-14, 2011.

Ebbensen, T.W., Lezec, H.J., Hiura, H., Bennett, J.W., Ghaemi, H.F., \& Thio, T. (1996). Electrical conductivity of individual carbon nanotubes. Nature, Vol. 382, No. 6586, (July 1996), pp. $54-56$.

EL Sabbagh, M.A, \& El-Ghazaly, S.M. (2009a). Miniaturized carbon nanotube-based RF resonator, in IEEE MTT-S Int. Microwave Symp. Dig., pp. 829-832, ISBN 978-1-42442803-8, Boston, MA, USA, June 7-12, 2009.

EL Sabbagh, M.A., El-Ghazaly, S.M., \& Naseem, H.A. (2009b). Carbon nanotube-based planar transmission lines. in IEEE MTT-S Int. Microwave Symp. Dig., pp. 353-356, ISBN 978-14244-2803-8, Boston, MA, USA, June 7-12, 2009. 
EL Sabbagh, M.A, \& El-Ghazaly, S.M. (2009c). Frequency-dependent circuit models of carbon nanotube networks, in Electrical Performance of Electronic Packaging and Systems, pp. 129-132, ISBN 978-1-4244-4447-2, Portland, OR, USA, October 19-21, 2009.

Fiske, T.J., Gokturk, H.S., \& Kaylon, D.M. (1997). Percolation in magnetic composites. Journal of Material Science, Vol.32, No. 20, (October 1997), pp. 5551-5560, ISSN 0022-2461.

Guo, N., DiBenedetto, S.A., Tewari, P., Lanagan, M.T., Ratner, M.A., \& Marks, T.J. (2010). Nanoparticle, size, shape, and interfacial effects on leakage current density, permittivity, and breakdown strength of metal oxide-polyolefin nanocomposites: experiment and theory. In Chemistry of Material, Vol. 22, No. 4, (February 2010), pp. 1567-1578.

Iijima, S. (1991). Helical microtubules of graphitic carbon. Nature, Vol. 354, (November 1991), pp. 56-58.

Jorio, A., Dresselhaus, M.S., \& Dresselhaus, G. (2008). Carbon Nanotubes: Advanced Topics in the Synthesis, Structure, Properties, and Applications, Springer, ISBN 978-3540728643, New York, USA.

Link, S., Mohamed, M.B., \& El-Sayed, M.A. (1999). Simulation of the optical Absorption spectra of gold nanorods as a function of their aspect ratio and the effect of the medium dielectric constant. Journal of Physical Chemistry B, Vol. 103, No. 16, (April 1999), pp. 3073-3077.

Liu, L., Matitsine, S., Gan, Y.B., Chen, L.F., \& Kong, L.B. (2007). Frequency dependence of effective permittivity of carbon nanotube composites. Journal of Applied Physics, Vol. 101, No. 9, (May 2007), pp. 094106-1-7, ISSN 0021-8979.

Meyyapan, M. (2005). Carbon Nanotubes: Science and Applications (1 ${ }^{\text {st }}$ ed.), CRC Press LLC, ISBN 9780849321115, Florida, USA.

O'Connell, M.J., (2006). Carbon Nanotubes Properties and Applications (1 $\left.1^{\mathrm{st}} \mathrm{ed}.\right)$, CRC Press LLC, ISBN 978-0849327483, Florida, USA.

Nelson, S.O. (1992). Estimation of permittivities of solids from measurements on pulverized or granular materials, In: Dielectric Properties of Heterogeneous Materials, A. Priou, Chap. 6, pp. 231-271, Elsevier, ISBN 9780444016461, New York, USA.

Ravindran, R., Gangopadhyay, K., Gangopadhyay, S., Mehta, N., \& Biswas, N. (2006). Permittivity enhancement of aluminum oxide thin films with the addition of silver nanoparticles. Applied Physics Letter, Vol. 89, No. 263511, (December 2006).

Saito, R., Dresselhaus, G., \& Dresselhaus, M.S. (1998). Physical Properties of Carbon Nanotubes, Imperial College Press, ISBN 978-1860942235, London.

Stauffer, D., \& Aharony, A. (1994). Introduction to Percolation Theory (2nd ed.), Taylor and Francis, ISBN 978-0748402533, Washington DC.

Tung, S., Rokadia, H., \& Li, W.J. (2007). A micro shear sensor based on laterally aligned carbon nanotubes. In Sensors and Actuators A: Physical, Vol. 133, No. 2, (February 2007), pp. 431-438.

Xu H., Anlage, S.M., Hu, L., \& Gruner, G. (2007). Microwave shielding of transparent and conducting single-walled carbon nanotube films. Applied Physics Letter, Vol. 90, No. 183119, (May 2007). 


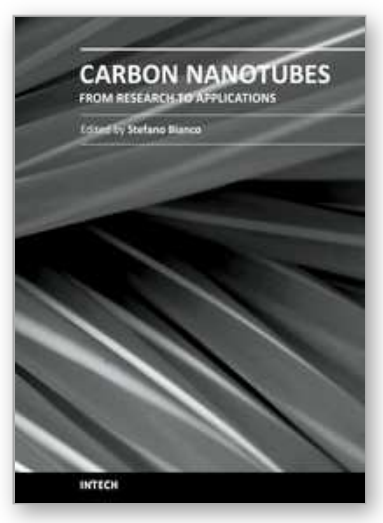

\author{
Carbon Nanotubes - From Research to Applications \\ Edited by Dr. Stefano Bianco
}

ISBN 978-953-307-500-6

Hard cover, 358 pages

Publisher InTech

Published online 20, July, 2011

Published in print edition July, 2011

Since their discovery in 1991, carbon nanotubes have been considered as one of the most promising materials for a wide range of applications, in virtue of their outstanding properties. During the last two decades, both single-walled and multi-walled CNTs probably represented the hottest research topic concerning materials science, equally from a fundamental and from an applicative point of view. There is a prevailing opinion among the research community that CNTs are now ready for application in everyday world. This book provides an (obviously not exhaustive) overview on some of the amazing possible applications of CNT-based materials in the near future.

\title{
How to reference
}

In order to correctly reference this scholarly work, feel free to copy and paste the following:

Emmanuel Decrossas and Samir. M. El-Ghazaly (2011). Microwave Dielectric Characterization of Carbon Nanotube Networks, Carbon Nanotubes - From Research to Applications, Dr. Stefano Bianco (Ed.), ISBN: 978953-307-500-6, InTech, Available from: http://www.intechopen.com/books/carbon-nanotubes-from-researchto-applications/microwave-dielectric-characterization-of-carbon-nanotube-networks

\section{INTECH}

open science | open minds

\section{InTech Europe}

University Campus STeP Ri

Slavka Krautzeka 83/A

51000 Rijeka, Croatia

Phone: +385 (51) 770447

Fax: +385 (51) 686166

www.intechopen.com

\section{InTech China}

Unit 405, Office Block, Hotel Equatorial Shanghai

No.65, Yan An Road (West), Shanghai, 200040, China

中国上海市延安西路65号上海国际贵都大饭店办公楼 405 单元

Phone: +86-21-62489820

Fax: +86-21-62489821 
(C) 2011 The Author(s). Licensee IntechOpen. This chapter is distributed under the terms of the Creative Commons Attribution-NonCommercialShareAlike-3.0 License, which permits use, distribution and reproduction for non-commercial purposes, provided the original is properly cited and derivative works building on this content are distributed under the same license. 